\title{
Sepsis: Pathophysiology and Treatment
}

\author{
Received: May 22, 2017; Accepted: May 26, 2017; Published: May 31, 2017
}

Few pathogens cause sepsis. The majority of sepsis causing bacteria is facultative anaerobes. This type of respiration is the most flexible and it facilitates pathogen survival, proliferation and dissemination in human tissues and blood. Other conditions for causing sepsis are thick slime layer and capsule, as well as production of antioxidant enzymes and substances (superoxide dismutase (SOD), catalase and glutathione) that protect bacteria against oxidative stress caused by reactive oxygen species of erythrocytes and leukocytes [1]. Pathogens need pore forming and membrane destroying enzymes and toxins for penetrating erythrocytes. Initial pathogen-host interaction occurs in the tissues where blood coagulation, resident and transmigrated leukocytes, platelets, complement proteins and NETs are the first line of innate antibacterial immunity. If bacteria survive in the tissues, they start to produce especially thick capsule and form biofilm. After overcoming the tissue barrier, pathogens enter the bloodstream and cause bacteremia. The latter transforms to sepsis when microbes survive oxidation on the surface of erythrocytes. Septic shock develops when bacteria enter erythrocytes, survive oxidation by oxygen released from oxyhemoglobin, proliferate inside erythrocytes and cause premature abundant release of oxygen from erythrocytes before their entering capillaries [2]. Premature release of oxygen has consequences: a) oxidation of blood plasma components (proteins, immune complexes, regulatory hormones, peptides, amino acids, lipids, vitamins and other substances necessary for cell nutrition, proliferation, protection, energy production, functioning, etc.); b) failure of oxygen delivery to cells and multi-organ hypoxia; c) disseminated intravascular coagulation with further massive haemorrhages because of consumption of blood coagulation factors. As a result, multiple organ dysfunctions develop [2].

At present the treatment of sepsis and septic shock is not pathogenesis-oriented. Available protocols include early antibiotic therapy, support of vital functions and correction of revealed abnormalities. As soon as bacteria enter erythrocytes and survive there, they become invulnerable because antibiotics and immune complexes poorly penetrate the membrane of erythrocytes [3]. Slime layer and thick capsule also protect pathogens against antimicrobials and host defence. Oxygen, prematurely released from erythrocytes, destroys the plasma components necessary for humoral regulation of tissues and cells and so the attempts to restore normal physiologic regulation may be futile. Oxygen therapy may increase the oxidation of plasma components and deteriorate the condition of patients. Simultaneously, tissue hypoxia gradually increases because the oxygen is prematurely
Hayk Minasyan

Private Laboratory, Department of
Immunology and Microbiology, Armenia

Corresponding author: Hayk Minasyan

haykminasyan@rambler.ru

Principal Researcher, Private Laboratory, Department of Immunology and Microbiology, Mamikonyanz, 38-38, Yerevan, Armenia.

Tel: 37477255295

Citation: Minasyan H. Sepsis: Pathophysiology and Treatment. J Intensive \& Crit Care 2017, 3:2.

released from erythrocytes to arterial blood and cannot be delivered to capillaries. Intoxication by metabolic wastes and bacterial toxins progresses, host mechanisms of excretion and neutralization fail and the fatal outcome in patients becomes inevitable.

The cornerstone of sepsis treatment should be the prevention of bacterial survival on the surface and inside erythrocytes by suppression of bacterial antioxidant defence. Inhibition of bacterial catalase, superoxide dismutase and glutathione production may restore bacteria killing by erythrocytes and prevent development of bacteremia to sepsis. Killed bacteria are digested in the liver and the spleen and bacteremia becomes selflimited. Inhibition of bacterial capsule and biofilm production can facilitate blood bacteria clearance by erythrocytes and increase the effectiveness of host immune complexes [4]. The inactivation of bacterial toxins may prevent the penetration of bacteria to erythrocytes.

The following is promising: 1 . toxin production inhibition by means of bacterial protein inhibition; 2 . toxin inactivation by binding with synthetic polymers, natural or synthetic antibodies, different toxin-inactivating compounds; 3 . toxin inactivation by modulation of target cell membrane characteristics [2]. Development of new antimicrobials is indispensable, although they will be effective in sepsis only in case of penetrating erythrocyte membranes, bacterial biofilm and capsules. The use of "biological weapon" (bacteriophage, Bdewillo like organisms, Saccharomyces) against sepsis causing bacteria may be useful in 
certain specific targets and cannot be universal [2]. Bloodstream bacteria removal by technical devices seems more promising.

The idea of bacteria removal from the bloodstream was presented more than 25 years ago by offering a special matrix of micro-encapsulated albumin activated charcoal (ACAC) [5]. Another device for removing bacterial toxins from blood was patented 10 years ago [6]. The device includes hollow fiber material for selective binding of the toxins and removing bacterial lipopolysaccharides (LPS) and lipoteichoic acids (LTA). Some years ago magnetic nanoparticles (MNPs) modified with bis-Zn-DPA (a synthetic ligand that binds bacteria) was tested [7]. An external device that mimics the structure of a spleen and cleanses the blood in acute sepsis has been constructed recently [8]. Blood flowing from a patient is mixed with magnetic nanobeads coated with an engineered human opsonin-mannosebinding lectin (MBL)-that captures a broad range of pathogens and toxins without activating complement factors or coagulation. Also a mechanical device has been developed to remove a variety of cytokines, lipopolysaccharide or C5a from plasma [9]. A prototype in-line filtration/adsorption device has been developed using novel synthetic pyrolysed carbon monoliths with controlled mesoporous domains of 2-50 nm [10]. A cytokine adsorption device $(C A D)$ filled with porous polymer beads which efficiently depletes middle-molecular weight cytokines from a circulating solution has been developed as well [11]. Continuous venovenous hemofiltration (CVVHF) combined with plasmapheresis (TPE) reduced mortality in single- and double-organ failure as high as $28 \%$ in septic patients [12]. At the same time, it should be taken into account that technical devices for removing bacteria, their products, cytokines and other substances do not affect the pathogenesis of sepsis and may be useless if bacteria have formed a reservoir in erythrocytes. 


\section{References}

1 Minasyan H (2016) Mechanisms and pathways for the clearance of bacteria from blood circulation in health and disease. Pathophysiology 23: 61-66.

2 Minasyan H (2017) Sepsis and septic shock: Pathogenesis and treatment perspectives. J Crit Care 40: 229-242.

3 Minasyan $\mathrm{H}$ (2014) Erythrocyte: Bacteria killer and bacteria pray. Int J Immunol 2: 1-7.

4 Minasyan H (2014) Erythrocyte and leukocyte: Two partners in bacteria killing. Int Rev Immunol 33: 490-497.

5 Marks DH, Medina F, Lee S, Blackmon A, Schuschereba ST (1988) Removal of bacteria from blood by charcoal hemoperfusion. Biomater Artif Cells Artif Organs 16: 135-140.

6 Siedel D, Boos K (2004) Device for removing bacterial toxins from blood or plasma, useful for treating sepsis, also for analysis and diagnosis, includes hollow fiber material for selective binding of the toxins. Patent DE10258944 (Germany).
7 Lee JJ, Jeong KJ, Hashimoto M, Kwon AH, Rwei A, et al. (2014) Synthetic ligand-coated magnetic nanoparticles for microfluidic bacterial separation from blood. Nano Lett 14: 1-5.

8 Kang JH, Super MI, Yung CW, Cooper RM, Domansky K, et al. (2014) An extracorporeal blood-cleansing device for sepsis therapy. Nature Med 20: 1211-1216.

9 Marshall JC (2008) Sepsis: Rethinking the approach to clinical research. J Leukoc Biol 83: 471-482.

10 Sandeman SR, Howell CA, Mikhalovsky SV, Phillips GJ, Lloyd AW, et al. (2008) Inflammatory cytokine removal by an activated carbon device in a flowing system. Biomaterials 29: 1638-1644.

11 DiLeo MV, Fisher JD, Burton BM, Federspiel WJ (2011) Selective improvement of tumor necrosis factor capture in a cytokine hemoadsorption device using immobilized anti-tumor necrosis factor. J Biomed Mater Res B Appl Biomater 96: 127-133.

12 Schmidt J, Mann S, Mohr VD, Lampert R, Firla U, et al. (2000) Plasmapheresis combined with continuous venovenous hemofiltration in surgical patients with sepsis. Intensive Care Med 26: 532. 